\title{
Los pacientes con placas coronarias vulnerables presentan mayores niveles séricos de metaloproteinasa-1
}

\author{
Patients with vulnerable coronary plaques have higher serum \\ metalloproteinase-1 levels
}

doi.org/10.23938/ASSN.0004

\section{G. Sánchez-Elvira ${ }^{1}$, I. Coma-Canella ${ }^{2}$, M. Artaiz ${ }^{2}$, J. Páramo ${ }^{3}$, J. Barba ${ }^{2}$, J. Calabuig ${ }^{2}$}

\begin{abstract}
RESUMEN
Fundamento. Las placas ateroscleróticas que producen la mayoría de los síndromes coronarios agudos al romperse son los fibroateromas de cápsula fina, denominados placas vulnerables. Éstas pueden ser detectadas únicamente con técnicas invasivas de imagen intracoronaria. Es preciso encontrar un biomarcador no invasivo que permita identificar a los pacientes con estas placas sin necesidad de cateterismo cardiaco. La metaloproteinasa-1 es una enzima involucrada en el metabolismo de la matriz extracelular que ha sido relacionada con la ruptura de las placas ateroscleróticas. Se desconocen sus niveles séricos en pacientes con placas vulnerables.
\end{abstract}

Material y métodos. Se incluyeron pacientes sometidos a cateterismo cardiaco por enfermedad coronaria estable. Se estudiaron las arterias coronarias con tomografía de coherencia óptica para detectar placas vulnerables. Se extrajeron muestras de sangre periférica y del seno coronario para analizar la concentración de metaloproteinasa-1.

Resultados. Se incluyeron 51 pacientes. Trece tenían al menos un fibroateroma de cápsula fina. No se encontraron diferencias significativas en las características clínicas, perfil lipídico ni proteína $\mathrm{C}$ reactiva entre los pacientes con y sin placas vulnerables. Los pacientes con placas vulnerables presentaron concentraciones significativamente mayores de metaloproteinasa-1, tanto en sangre periférica ( $7330 \pm 5541$ vs $2894 \pm 1783 \mathrm{pg} / \mathrm{ml}, \mathrm{p}=0,025)$ como en seno coronario $(6012 \pm 3854$ vs $2707 \pm 1252 \mathrm{pg} / \mathrm{ml}, \mathrm{p}=0,047)$.

Conclusiones. Los pacientes con placas vulnerables presentaron niveles séricos significativamente mayores de metaloproteinasa-1. Se requieren estudios con seguimiento clínico para evaluar el valor pronóstico de la metaloproteinasa-1 sérica.

Palabras clave: Placa vulnerable. Fibroateroma de cápsula fina. Biomarcador. Metaloproteinasa-1. Síndrome coronario agudo. \begin{abstract}
Background. Most acute coronary syndromes are caused by the fracture of a vulnerable atherosclerotic plaque. These plaques are thin cap fibroatheromas, which can only be detected with invasive coronary imaging techniques. It is necessary to find a non-invasive biomarker of these vulnerable plaques in order to identify patients at risk without a coronary angiography. Metalloproteinase-1 is an enzyme involved in extracellular matrix metabolism which has been correlated with the rupture of atherosclerotic plaques. Its serum levels in patients with vulnerable plaques remain unknown.
\end{abstract}

Methods. Patients with suspected stable coronary artery disease undergoing coronary angiography in our hospital were included. The coronary arteries were studied with optical coherence tomography to detect vulnerable plaques. Blood samples were taken from a peripheral vein and from the coronary sinus, to assess metalloproteinase-1 levels.

Results. Fifty-one patients were included, 13 of whom had at least one vulnerable plaque. There were not significant differences in clinical characteristics, lipid profile or C reactive protein levels, between patients with or without vulnerable plaques. Patients with vulnerable plaques had significant higher metalloproteinase-1 levels both in peripheral $(7330 \pm 5541$ vs $2894 \pm 1783 \mathrm{pg} / \mathrm{ml}, \mathrm{p}=0.025)$ and coronary sinus serum $(6012 \pm 3854$ vs $2707 \pm 1252 \mathrm{pg} / \mathrm{ml}$, $\mathrm{p}=0.047$ ).

Conclusions. Patients with vulnerable plaques had significantly higher metalloproteinase-1 serum levels. Further studies with clinical follow up are needed to assess the prognostic value of serum metalloproteinase-1.

Keywords: Vulnerable plaque. Thin cap fibroatheroma. Biomarker. Metalloproteinase-1. Acute coronary syndrome.
1. Servicio de Cardiología. Complejo Hospitalario de Navarra. Pamplona.

2. Departamento de Cardiología. Clínica Universidad de Navarra. Pamplona.

3. Departamento de Hematología. Clínica Universidad de Navarra. CIMA. Pamplona.

Recepción: 29/07/2016

Aceptación provisional: 13/12/2016

Aceptación definitiva: 16/02/2017

\section{Correspondencia:}

Guillermo Sánchez-Elvira

Servicio de Cardiología

Complejo Hospitalario de Navarra

Irunlarrea, 3.

31008 Pamplona.

Email: sanchez-elvira@hotmail.com

Becas: Este trabajo se financió parcialmente con una beca del Departamento de Salud, Gobierno de Navarra (ref 15/2008) y del Plan de Investigación de la Universidad de Navarra (PIUNA). 


\section{INTRODUCCIÓN}

La revascularización coronaria precoz y el uso de nuevos fármacos han disminuido la mortalidad y morbilidad de los pacientes con síndrome coronario agudo (SCA). Estos avances se han centrado en el tratamiento rápido e invasivo de los SCA cuando ya han tenido lugar, pero sería mucho más eficaz prevenirlos.

Los factores de riesgo clásicos no son suficientes para distinguir qué pacientes van a presentar un SCA en un futuro próxi$\mathrm{mo}^{1}$. De hecho, una proporción importante de los eventos los presentan pacientes con los factores de riesgo controlados, o incluso sin factores de riesgo, por lo que son necesarios mejores marcadores de vulnerabilidad que identifiquen a los pacientes realmente en riesgo.

Los estudios anatomopatológicos han mostrado que la principal lesión culpable de los SCA es la rotura de una placa aterosclerótica coronaria vulnerable. Estas placas se caracterizan por presentar un remodelado positivo, un núcleo lipídico grande con core necrótico significativo (producto de la lisis de macrófagos saturados de grasas) y un infiltrado linfocitario ${ }^{2}$. Las placas ateroscleróticas con estas características pero que no están rotas, con una cápsula fibrosa fina $(<65 \mu \mathrm{m})$, se denominan fibroateromas de cápsula fina (TCFA) $)^{3,4}$ o placas vulnerables. Su presencia es un factor predictivo independiente para presentar un $\mathrm{SCA}^{5}$.

Los TCFA han sido estudiados in vivo con técnicas de imagen intravascular, como la tomografía de coherencia óptica (OCT), cuya precisión permite medir el grosor de la cápsula fibrosa. Estas técnicas de imagen son invasivas y requieren cateterismo cardiaco. Disponer de un marcador no invasivo de la presencia de TCFA sería de gran utilidad para identificar a pacientes de alto riesgo sin la necesidad de un cateterismo. Estos pacientes podrían beneficiarse de un tratamiento médico más intensivo o incluso de intervencionismo coronario percutáneo (ICP) $)^{6,7}$.

Las placas rotas, responsables de los SCA, contienen altos niveles de metalopro- teinasas (MMP), que están involucradas en el metabolismo de la matriz extracelular, pueden debilitar la cápsula fibrosa y producir su rotura ${ }^{8}$. La metaloproteinasa-1 (MMP-1) tiene como principal sustrato al colágeno tipo I, responsable de la fuerza elástica de los tejidos. Se ha demostrado una gran expresión histológica de MMP-1 en placas ateroscleróticas carotídeas y coronarias, sobre todo en aquellas que estaban rotas ${ }^{9,10}$. También se han correlacionado sus niveles en sangre circulante con la presencia de enfermedad coronaria ${ }^{11,12}$.

El objetivo de este estudio es evaluar los niveles séricos de MMP-1 en pacientes con y sin placas vulnerables, detectadas mediante OCT.

\section{MATERIAL Y MÉTODOS}

Se realizó un estudio de casos y controles prospectivo, unicéntrico. Se cumplieron todos los requisitos actualizados de la declaración de Helsinki. El comité ético de la Clínica Universidad de Navarra aprobó el protocolo del estudio y todos los pacientes incluidos dieron su consentimiento informado.

Se incluyeron pacientes con sospecha de enfermedad coronaria estable sometidos a cateterismo cardiaco en la Clínica Universidad de Navarra. Los criterios de exclusión fueron la edad menor de 18 años, la insuficiencia renal mayor que ligera (filtrado glomerular $<50 \mathrm{ml} / \mathrm{min}$ ) y el SCA.

La técnica de adquisición de imagen intravascular en este estudio ya ha sido publicada previamente ${ }^{13}$. Se utilizó un acceso arterial femoral 6 French. Tras la angiografía coronaria se avanzó el catéter de OCT en las 3 arterias coronarias, de forma secuencial. Se obtuvieron las imágenes con la retirada automática del catéter mientras se inyectaba contraste yodado en la coronaria para evitar la interferencia de la sangre. El análisis de la OCT fue realizado de forma separada por dos cardiólogos intervencionistas, de acuerdo a criterios establecidos ${ }^{14}$. Las placas ateroscleróticas con una señal de OCT hipointensa, con bordes mal definidos, fue diagnóstica de 
núcleo lipídico. Cuando éste estaba presente en $>2$ cuadrantes de la imagen axial de la placa, ésta se definió como placa lipídica. La cápsula fibrosa se identificó como una señal intensa y homogénea cubriendo el contenido lipídico, separándolo de la luz del vaso ${ }^{15,16}$. Su grosor se midió 3 veces en cada corte axial y se tomó la media. El TCFA se definió como una placa lipídica con una cápsula fibrosa $<65 \mu \mathrm{m}$ en su parte más fina (Fig. 1).

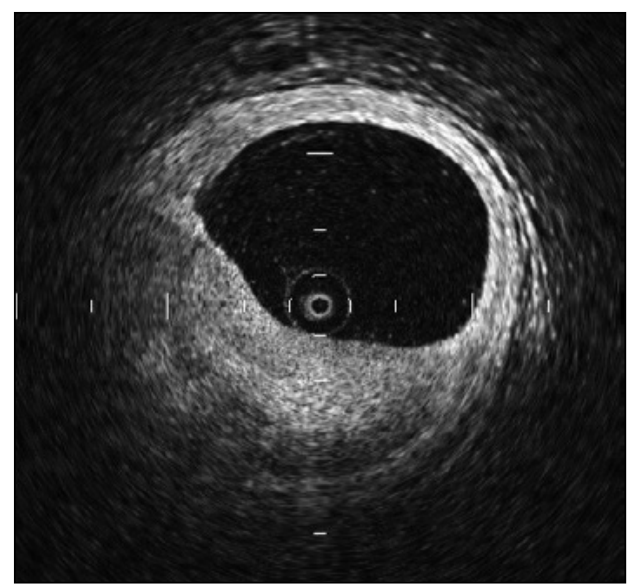

Figura 1. Una placa vulnerable: placa lipídica que ocupa más de 2 cuadrantes, con una cápsula fibrosa menor de $65 \mu \mathrm{m}$.

Las muestras de sangre periférica de los pacientes se extrajeron en la sala de hemodinámica inmediatamente antes del cateterismo cardiaco. Al finalizar el estudio coronario y tras ICP en los pacientes que lo requirieron, se cateterizó desde un acceso venoso femoral el seno coronario con un catéter Cournand, extrayendo así las muestras de sangre del retorno venoso coronario. No hubo complicaciones de acceso vascular ni nefropatías por contraste en ninguno de los pacientes.

Los niveles séricos de MMP-1 se midieron en ambos tipos de muestra con un kit comercial de inmunoensayo ligado a enzimas (ELISA) (Millipore's MILLIPLEXTM Human MMP Panel 2; Millipore Corp, Missouri, USA). El kit tiene un coeficiente de variación intraensayo del $2,6 \%$ e inter-ensayo del $8,4 \%$.

\section{Análisis estadístico}

Las variables continuas se expresaron como media \pm desviación estándar. Las variables categóricas como número y porcentaje. Se comprobó la normalidad de la distribución de las variables con el test de Shapiro-Wilk. Las comparaciones se realizaron con el test de Chi cuadrado o la t de Student según la variable. Las variables no paramétricas se analizaron con el test exacto de Fisher o el de Wilcoxon-MannWhitney. Todos los valores de $\mathrm{p}$ fueron bilaterales, con una significación estadística fijada en $<0,05$. El análisis estadístico se realizó con el programa SPSS 20.0 (SPSS, Inc).

\section{RESULTADOS}

Se incluyeron en el estudio un total de 51 pacientes. Se excluyeron posteriormente del análisis 6 pacientes por un problema técnico con el archivo del estudio de OCT $(n=1)$, por mala conservación de la muestra de sangre $(n=1)$ y por estudio incompleto por alta complejidad del ICP a realizar $(n=4)$. Se estudiaron un total de 93 arterias (2,06 $\pm 0,72$ vasos por paciente). Se estudiaron las tres arterias coronarias en 13 pacientes ( $28,9 \%$ de los pacientes), dos arterias en 22 pacientes $(48,9 \%)$ y solo una arteria en 10 pacientes (22,2\%). Las razones por las que no se estudiaron todas las arterias fueron: a) 32 arterias eran angiográficamente normales (sin placas visibles por angiografía); b) 6 arterias eran demasiado finas para el paso del catéter de OCT; c) 4 arterias eran de un calibre demasiado grande para el alcance de la OCT.

Se identificaron 128 placas ateroscleróticas (2,84 placas/paciente). Catorce placas cumplían criterios de TCFA (10,9\% del total de placas). Trece pacientes presentaban al menos un TCFA (28,9\% de los pacientes). Un paciente tenía dos TCFA. Los TCFA estaban localizados en diferentes segmentos de las tres arterias coronarias, sin una tendencia significativa por ninguno de ellos. La media del grosor de la cápsula fibrosa en los TCFA fue de $51,6 \pm 8 \mu \mathrm{m}$. 
No hubo diferencias en las características clínicas y parámetros bioquímicos de los pacientes con y sin TCFA, que se resumen en las tablas 1 y 2 respectivamente. El uso de estatinas, betabloqueantes, antagonistas de la enzima convertidora de angio- tensina o antagonistas de los receptores tipo 1 de la angiotensina II en el momento de la inclusión fue de aproximadamente el $50 \%$ para cada uno de los fármacos, sin diferencias entre los dos grupos.

Tabla 1. Características clínicas

\begin{tabular}{|c|c|c|c|}
\hline & $\begin{array}{c}\text { Sin TCFA } \\
(n=32)\end{array}$ & $\begin{array}{c}\text { Con TCFA } \\
(n=13)\end{array}$ & $\boldsymbol{P}$ \\
\hline Edad (años) & $61 \pm 9$ & $65 \pm 7$ & 0,09 \\
\hline Sexo (masculino), n (\%) & $24(75)$ & $12(92)$ & 0,41 \\
\hline Índice de masa corporal $\left(\mathrm{kg} / \mathrm{m}^{2}\right)$ & $29 \pm 4$ & $30 \pm 5$ & 0,52 \\
\hline \multicolumn{4}{|l|}{ Factores de riesgo, n (\%): } \\
\hline Tabaquismo & $20(63)$ & $11(85)$ & 0,06 \\
\hline Hipertensión & $22(69)$ & $8(62)$ & 0,57 \\
\hline Hipercolesterolemia & $4(31)$ & $9(69)$ & 0,49 \\
\hline Diabetes mellitus & $12(38)$ & $7(54)$ & 0,71 \\
\hline Antecedentes Familiares de EAC & $8(25)$ & $2(15)$ & 0,67 \\
\hline SCA previo, n (\%) & $4(13)$ & $5(39)$ & 0,07 \\
\hline Estatina, n (\%) & $21(66)$ & $9(69)$ & 0,68 \\
\hline Betabloqueante, n (\%) & $13(41)$ & $6(46)$ & 0,75 \\
\hline IECA, n (\%) & $9(28 \%)$ & $2(15)$ & 0,62 \\
\hline ARAII, n (\%) & $6(19 \%)$ & $5(39)$ & 0,34 \\
\hline
\end{tabular}

Valores presentados como media \pm desviación estándar o número (porcentaje).

TCFA: fibroateroma de cápsula fina; EAC: enfermedad arterial coronaria; IECA: inhibidor de la enzima convertidora de angiotensina; ARAII: antagonista de los receptores de la angiotensina; SCA: síndrome coronario agudo.

Tabla 2. Parámetros bioquímicos

\begin{tabular}{lccc}
\hline & $\begin{array}{c}\text { Sin TCFA } \\
(\boldsymbol{n}=\mathbf{3 2})\end{array}$ & $\begin{array}{c}\text { Con TCFA } \\
(\boldsymbol{n}=\mathbf{1 3})\end{array}$ & $\boldsymbol{P}$ \\
\hline $\begin{array}{l}\text { Perfil lipídico: } \\
\text { LDL (mg/dl) } \\
\text { HDL (mg/dl) }\end{array}$ & $104 \pm 42.0$ & $82.9 \pm 26.1$ & 0.18 \\
\cline { 2 - 4 } Triglicéridos (mg/dl) & $56 \pm 27.0$ & $44.9 \pm 12.4$ & 0.14 \\
\hline Hemoglobina Alc (\%) & $119 \pm 60.8$ & $93.5 \pm 32.9$ & 0.15 \\
\hline PCR ultrasensible (mg/dl) & $6.6 \pm 1.2$ & $6.8 \pm 1.6$ & 0.72 \\
\hline Creatinina (mg/dl) & $0.41 \pm 0.3$ & $0.31 \pm 0.28$ & 0.56 \\
\hline
\end{tabular}

Valores presentados como media \pm desviación estándar.

TCFA: fibroateroma de cápsula fina; LDL: lipoproteína de baja densidad; HDL: lipoproteína de alta densidad; PCR: proteína $\mathrm{C}$ reactiva. 
Los pacientes con TCFA presentaron una concentración sérica mayor de MMP-1 que los pacientes sin TCFA, tanto en sangre periférica $(7.330 \pm 5.541$ vs $2.894 \pm 1.783 \mathrm{pg} /$ $\mathrm{ml}, \mathrm{p}=0,025)$ como en sangre del seno coronario $(6.012 \pm 3.854$ vs $2.707 \pm 1.252 \mathrm{pg} / \mathrm{ml}$, $\mathrm{p}=0,047$ ) (Fig. 2 y 3 ).

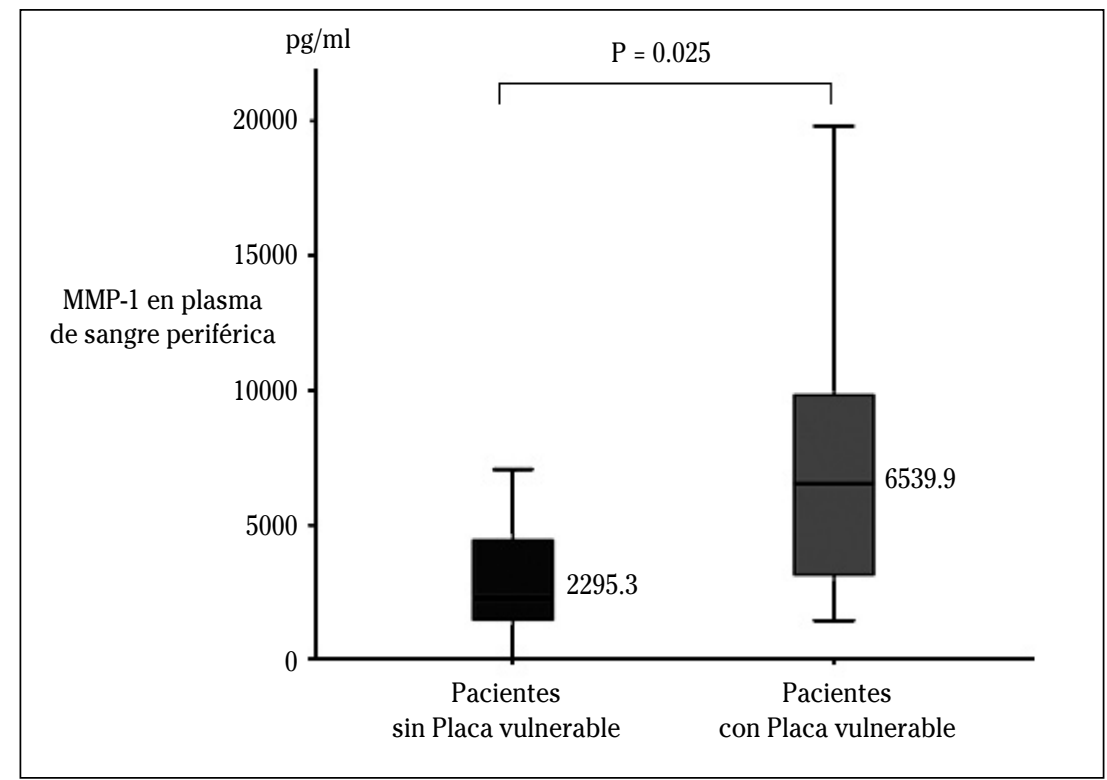

Figura 2. Comparación de los niveles séricos de metaloproteinasa-1 en sangre periférica.

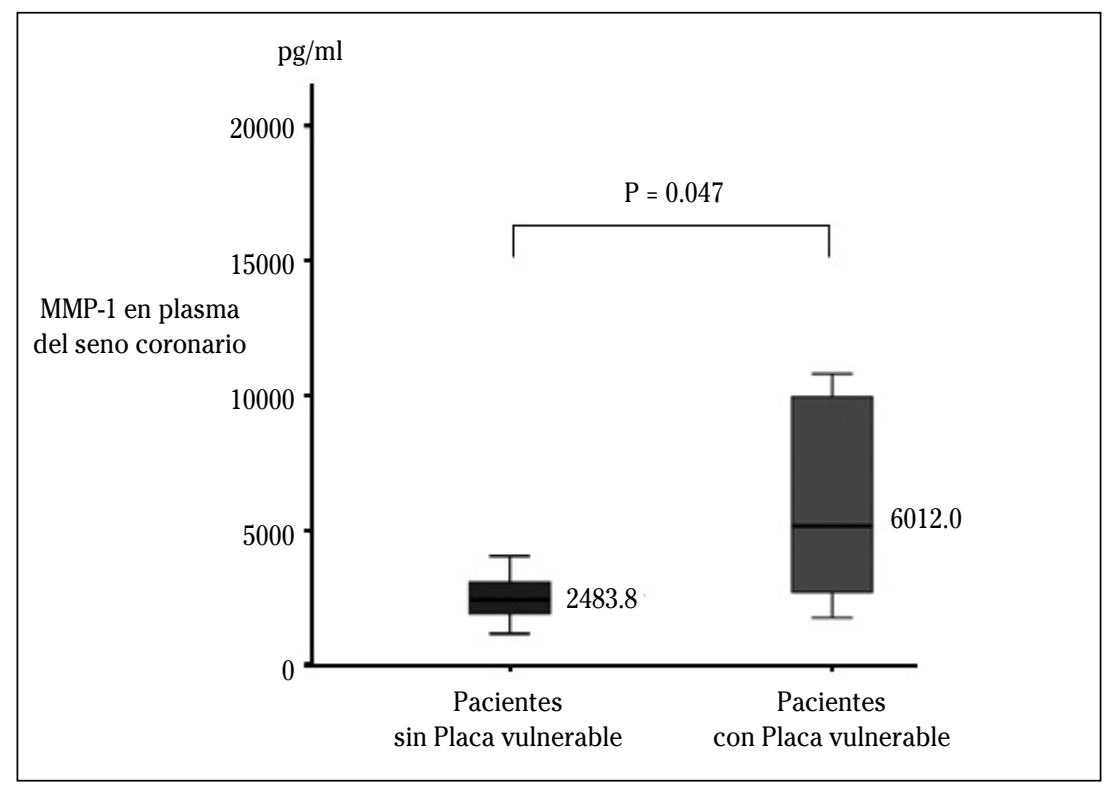

Figura 3. Comparación de los niveles séricos de metaloproteinasa-1 en sangre del seno coronario. 


\section{DISCUSIÓN}

Este es el primer estudio que evalúa los niveles séricos de MMP-1 en pacientes con placas coronarias vulnerables.

En el estudio se incluyeron pacientes con enfermedad coronaria estable. Se han realizado varios estudios de imagen intracoronaria para detectar placas vulnerables en pacientes con SCA, pero los marcadores que detectemos en un SCA serán marcadores diagnósticos de SCA, no marcadores de paciente en riesgo. Un biomarcador útil para prevenir los SCA debe poder detectarse en sangre cuando no hay placas rotas, por lo que el estudio de posibles biomarcadores tiene que realizarse en pacientes estables.

Los TCFA son considerados la forma más habitual de placa vulnerable, cuya ruptura causa los $\mathrm{SCA}^{2-4}$. Se han estudiado con diferentes técnicas de imagen intracoronaria, como el ultrasonido intravascular (IVUS), la histología virtual (HV) y la tomografía de coherencia óptica (OCT) ${ }^{13-17}$. En el estudio PROSPECT se identificaron TCFA con IVUS e HV (que mejora la sensibilidad del IVUS para detectar núcleos lipídicos ${ }^{18}$ ). En el seguimiento de los pacientes no hubo más SCA en los que tenían TCFA ${ }^{19}$. Estos resultados se explican por la falta de sensibilidad y especificidad del IVUS y la HV para identificar placas realmente vulnerables, porque no pueden diferenciar entre fibroateromas con cápsula gruesa o fina ${ }^{20}$. Además, se ha puesto en entredicho la capacidad de la HV para detectar el core necrótico ${ }^{21}$. La gran precisión de la OCT para medir el grosor de la cápsula fibrosa la convierten en la mejor técnica de imagen intravascular para detectar placas vulnerables $^{22,23}$.

Estas técnicas de imagen son invasivas, con el riesgo potencial y el coste que implica. Un biomarcador plasmático que identificara a pacientes con TCFA permitiría un cribado poblacional con la extracción de una muestra de sangre.

Entre los posibles candidatos a biomarcadores de riesgo coronario están las MMP. Son proteasas involucradas en el metabolismo de la matriz extracelular y han sido relacionadas con el adelgazamiento progresivo y rotura de las cápsulas fibrosas de los TCFA $^{24-26}$.

Otros grupos y el nuestro, han objetivado niveles aumentados de MMP-1 tanto in vitro como in vivo como marcador de aterosclerosis ${ }^{27-29}$. En placas carotídeas la expresión histológica de MMP-1 es mayor en los bordes de los núcleos lipídicos, junto a la cápsula fibrosa, que es donde normalmente se rompen las placas ${ }^{9}$. También se ha descrito una fuerte correlación entre el porcentaje de núcleo lipídico ocupado por hemorragia y el porcentaje del perímetro del núcleo lipídico positivo para MMP- ${ }^{9}$. Morgan y col demostraron mayor expresión genética de MMP-1 en placas con cápsula fina ${ }^{30}$. Guo y col describieron mayor concentración de MMP-1 en el tejido de placas rotas frente a las no rotas ${ }^{10}$. Todos estos estudios encontraron asociación entre la presencia de aterosclerosis, placas rotas o con cápsula adelgazada, con los niveles de MMP-1, pero a nivel histológico.

También se ha descrito que algunos haplotipos de MMP-1 se asocian a mayor incidencia de $\mathrm{SCA}^{31}$.

Se ha encontrado una buena correlación entre niveles altos de MMP-1 en sangre periférica y la presencia de enfermedad coronaria ${ }^{11,12}$. Estos estudios correlacionaron MMP-1 sérica con la presencia de lesiones coronarias, pero no específicamente con TCFA.

En el presente estudio se ha evaluado la correlación entre los niveles de MMP-1 en plasma y la presencia de TCFA. La invasividad del estudio, que incluyó la OCT de las 3 arterias coronarias y la extracción de sangre del seno coronario, limita el número de pacientes incluidos. El haber encontrado diferencias significativas en la concentración de MMP-1 en una muestra limitada de pacientes resulta muy prometedor. Los resultados muestran una importante dispersión en el grupo de pacientes con placas vulnerables, por el hecho de ser un grupo con menos individuos. No hubo diferencias significativas en las características clínicas ni bioquímicas basales entre los pacientes con o sin TCFA que pudieran explicar estas diferencias. 
Se midieron los niveles de MMP-1 en sangre del seno coronario, que es el retorno venoso de las coronarias. En caso de haber un origen extracardiaco de MMP-1 se habría podido detectar la presencia de un gradiente significativo, con mayores niveles en sangre periférica. Tampoco se encontraron mayores niveles en el seno coronario respecto a sangre periférica. Esto puede ser debido al número limitado de sujetos incluidos, que no haya permitido encontrar un gradiente estadísticamente significativo o a que la degradación de la MMP-1 en sangre no sea suficientemente rápida como para generar dicho gradiente, igualándose las concentraciones en todos los territorios vasculares.

En conclusión, los niveles séricos de MMP-1 tanto de sangre periférica como del seno coronario fueron significativamente mayores en los pacientes con TCFA. Esto sugiere que la MMP-1 podría ser un biomarcador que identifique pacientes con mayor riesgo de presentar un SCA. Se requieren más estudios con seguimiento clínico para confirmar el valor predictivo de los niveles altos de MMP-1 en sangre periférica.

\section{BIBLIOGRAFÍA}

1. Khot UN, Кнот MB, Bajzer CT, Sapp SK, Ohman EM, Brener SJ et al. Prevalence of conventional risk factors in patients with coronary heart disease. JAMA 2003; 290: 898-904.

2. Burke AP, Farb A, Malcom GT, Liang YH, Smialek J, VIRMANI R. Coronary risk factors and plaque morphology in men with coronary disease who died suddenly. N Engl J Med 1997; 336: 1276-1282.

3. Kolodgie FD, Virmani R, Burke AP, Farb A, WeBER DK, KUTYs R et al. Pathologic assessment of the vulnerable human coronary plaque. Heart 2004; 90: 1385-1391.

4. Virmani R, Burke AP, Farb A, Kolodgie FD. Pathology of the vulnerable plaque. J Am Coll Cardiol 2006; 47: C13-18.

5. Cheng JM, García-García HM, de Boer SP, Kardys I, Heo JH, AKKERHUIS KM et al. In vivo detection of high-risk coronary plaques by radiofrequency intravascular ultrasound and cardiovascular outcome: results of the ATHEROREMO-IVUS study. Eur Heart J 2014; 35: 639-647.
6. LINDAHL B. Are there really biomarkers of vulnerable plaque? Clin Chem 2012; 58: 151-153.

7. Fuster V, Moreno PR, Fayad ZA, Corti R, Badimon JJ. Atherothrombosis and high-risk plaque: part I: evolving concepts. J Am Coll Cardiol 2005; 46: 937-954.

8. Newby AC. Metalloproteinases promote plaque rupture and myocardial infarction: $A$ persuasive concept waiting for clinical translation. Matrix Biol 2015; 44-46: 157-166.

9. Nikkari ST, O’Brien KD, Ferguson M, Hatsukami T, Welgus HG, AlPERs CE et al. Interstitial collagenase (MMP-1) expression in human carotid atherosclerosis. Circulation 1995; 92: 13931398.

10. Guo A, Wei L, Shi H, Li X, You L. [Matrix metalloproteinase-1 and coronary atheroslerotic plaque rupture]. Zhonghua Bing Li Xue Za Zhi 2000; 29: 263-266.

11. Lehrke M, Greif M, Broedl UC, Lebherz C, LaUBENDER RP, BECKER A et al. MMP-1 serum levels predict coronary atherosclerosis in humans. Cardiovasc Diabetol 2009; 8: 50.

12. Tanindi A, Sahinarslan A, Elbeg S, Cemri M. Relationship between MMP-1, MMP-9, TIMP-1, IL-6 and risk factors, clinical presentation, extent and severity of atherosclerotic coronary artery disease. Open Cardiovasc Med J 2011; 5: 110-116.

13. Sánchez-Elvira G, Coma-Canella I, Artaiz M, PÁramo JA, Barba J, Calabuig J. Characterization of coronary plaques with combined use of intravascular ultrasound, virtual histology and optical coherence tomography. Heart Int 2010; 5: e12.

14. Jang IK, Tearney GJ, MacNeill B, Takano M, MoselewsKi $\mathrm{F}$, IfTIMA $\mathrm{N}$ et al. In vivo characterization of coronary atherosclerotic plaque by use of optical coherence tomography. Circulation 2005; 111: 1551-1555.

15. Yabushita H, Bouma BE, Houser SL, Aretz HT, JANG IK, SchlENDORF KH et al. Characterization of human atherosclerosis by optical coherence tomography. Circulation 2002; 106: 1640-1645.

16. SaWada T, Shite J, García-García HM, Shinke T, Watanabe S, OtAKe $\mathrm{H}$ et al. Feasibility of combined use of intravascular ultrasound radiofrequency data analysis and optical coherence tomography for detecting thin-cap fibroatheroma. Eur Heart J 2008; 29: 1136-1146.

17. Jang IK, Bouma BE, Kang DH, Park SJ, Park SW, SEUNG KB et al. Visualization of coronary atherosclerotic plaques in patients using optical coherence tomography: comparison with intravascular ultrasound. J Am Coll Cardiol 2002; 39: 604-609. 
18. Rodriguez-Granillo GA, García-García HM, Mc FAdDen EP, Valgimigli M, AOKI J, DE Feyter P et al. In vivo intravascular ultrasound-derived thin-cap fibroatheroma detection using ultrasound radiofrequency data analysis. J Am Coll Cardiol 2005; 46: 2038-2042.

19. Stone GW, Maehara A, Lansky AJ, de Bruyne B, Cristea E, Mintz GS et al. A prospective natural-history study of coronary atherosclerosis. N Engl J Med 2011; 364: 226-235.

20. Low AF, Kawase Y, Chan YH, Tearney GJ, Bouma $\mathrm{BE}, \mathrm{J}_{\mathrm{ANG}} \mathrm{IK}$. In vivo characterisation of coronary plaques with conventional grey-scale intravascular ultrasound: correlation with optical coherence tomography. EuroIntervention 2009; 4: 626-632.

21. Sales FJ, Falcão BA, Falcão JL, Ribeiro EE, Perin MA, HoRTA PE et al. Evaluation of plaque composition by intravascular ultrasound "virtual histology": the impact of dense calcium on the measurement of necrotic tissue. Eurolntervention 2010; 6: 394-399.

22. Barlis P, Serruys PW, Gonzalo N, van der Giessen WJ, DE JAEgere PJ, Regar E. Assessment of culprit and remote coronary narrowings using optical coherence tomography with long-term outcomes. Am J Cardiol 2008; 102: 391-395.

23. Raffel OC, Merchant FM, Tearney GJ, Chia S, Gauthier DD, Pomerantsev E et al. In vivo association between positive coronary artery remodelling and coronary plaque characteristics assessed by intravascular optical coherence tomography. Eur Heart J 2008; 29: 1721-1728.

24. Shah PK, Falk E, Badimon JJ, Fernandez-Ortiz A, Mailhac A, Villareal-Levy G et al. Human monocyte-derived macrophages induce collagen breakdown in fibrous caps of atherosclerotic plaques. Potential role of matrix-degrading metalloproteinases and implications for plaque rupture. Circulation 1995; 92: 1565-1569.
25. Higashikata T, Yamagishi M, Higashi T, Nagata I, IIHARA K, MiYAmoto S et al. Altered expression balance of matrix metalloproteinases and their inhibitors in human carotid plaque disruption: results of quantitative tissue analysis using real-time RT-PCR method. Atherosclerosis 2006; 185: 165-172.

26. Yu Y, Koike T, Kitajima S, Liu E, Morimoto M, SHIOMI $\mathrm{M}$ et al. Temporal and quantitative analysis of expression of metalloproteinases (MMPs) and their endogenous inhibitors in atherosclerotic lesions. Histol Histopathol 2008; 23: 1503-1516.

27. Páramo JA, Orbe J, Fernández J. Fibrinolysis/ proteolysis balance in stable angina pectoris in relation to angiographic findings. Thromb Haemost 2001; 86: 636-639.

28. Orbe J, Rodríguez JA, Arias R, Belzunce M, NesPEREIRA B, PÉrez-Ilzarbe M et al. Antioxidant vitamins increase the collagen content and reduce MMP-1 in a porcine model of atherosclerosis: implications for plaque stabilization. Atherosclerosis 2003; 167: 45-53.

29. Orbe J, Fernández L, Rodríguez JA, Rábago G, Belzunce M, Monasterio A et al. Different expression of MMPs/TIMP-1 in human atherosclerotic lesions. Relation to plaque features and vascular bed. Atherosclerosis 2003; 170: 269-276.

30. Morgan AR, Rerkasem K, Gallagher PJ, Zhang B, Morris GE, CALDER PC et al. Differences in matrix metalloproteinase-1 and matrix metalloproteinase-12 transcript levels among carotid atherosclerotic plaques with different histopathological characteristics. Stroke 2004; 35: 1310-1315.

31. Pearce E, Tregouet DA, Samnegård A, Morgan AR, Cox C, Hamsten A et al. Haplotype effect of the matrix metalloproteinase-1 gene on risk of myocardial infarction. Circ Res 2005; 97: 1070-1076. 